\title{
Sulbactam Enhances in vitro Activity of $\beta$-Lactam Antibiotics Against Acinetobacter baumannii
}

\author{
Leilei Wang ${ }^{1,2}$ \\ Yuancheng Chen ${ }^{1-3}$ \\ Renru Han ${ }^{1,2}$ \\ Zhiwei Huang ${ }^{1-3}$ \\ Xuefei Zhang ${ }^{1,2}$ \\ Fupin $\mathrm{Hu}^{1,2}$ \\ Fan Yang ${ }^{1,2}$
}

'Institute of Antibiotics, Huashan Hospital, Fudan University, Shanghai, People's Republic of China; ${ }^{2}$ Key Laboratory of Clinical Pharmacology of Antibiotics, Ministry of Health, Shanghai, People's Republic of China; ${ }^{3}$ Phase I Clinical Research Center, Huashan Hospital, Fudan University, Shanghai, People's Republic of China
Correspondence: Fan Yang; Fupin Hu Institute of Antibiotics, Huashan Hospital, Fudan University, No. 12 Middle

Wulumuqi Road, Shanghai, 200040,

People's Republic of China

Tel +862152888193

Email fanyang9@fudan.edu.cn;

hufupin@fudan.edu.cn
Purpose: To evaluate in vitro activities of $\beta$-lactam antibiotics alone and in combination with sulbactam at different ratios against Acinetobacter baumannii clinical strains from China.

Methods: A total of 300 clinical isolates of $A$. baumannii were collected from 29 hospitals across China in 2018. Susceptibility to common antibiotics was assessed, and $\beta$-lactamase genes were detected. In vitro activity of ampicillin, cefoperazone and imipenem was tested alone and in combination with sulbactam at the ratios of $2: 1,1: 1,1: 1.5,1: 2,1: 2.5$ and 1:3. Results: High resistant rates for common antibiotics were observed except tigecycline and polymyxin B. Among carbapenem-resistant A. baumannii, 97.3\% isolates harbored $b l a_{\mathrm{OXA}-23}$. $\mathrm{MIC}_{50}$ and $\mathrm{MIC}_{90}$ values for sulbactam were $32 \mathrm{mg} / \mathrm{L}$ and $64 \mathrm{mg} / \mathrm{L}$, respectively. High resistant rates for ampicillin, cefoperazone and imipenem were observed $(92.3 \%, 93 \%$ and $85.3 \%$, respectively). A stepwise increase in the ratio of sulbactam to partner $\beta$-lactam antibiotics led to a stepwise decrease in the MICs and a stepwise increase in the susceptible rates. The susceptible rates for imipenem-sulbactam 1:3, ampicillin-sulbactam 1:3 and cefoperazone-sulbactam 1:3 reached $16.3 \%, 58.3 \%$ and $91 \%$, respectively.

Conclusion: The increasing proportion of sulbactam could enhance antimicrobial activities of imipenem-sulbactam, ampicillin-sulbactam and cefoperazone-sulbactam combinations against $A$. baumannii clinical strains in China, with cefoperazone-sulbactam as the most potent compound.

Keywords: sulbactam, ampicillin, cefoperazone, imipenem, Acinetobacter baumannii

\section{Introduction}

Acinetobacter baumannii is one of the most troublesome pathogens among healthcare-associated infections, and it could evolve into multidrug-resistance (MDR) clones, even extensively-drug resistance (XDR) clones, which are often associated with prolonged length and increased cost of hospital stay as well as high mortality. ${ }^{1}$ Apparently, intrinsic resistance and acquired resistance due to inappropriate administration of antimicrobial agents could be the critical reasons for the development of MDR bacteria. ${ }^{2,3}$

Carbapenem-resistant A. baumannii (CRAB), as a matter of great concern, has been labelled as "critical pathogen" by World Health Organization (WHO) and as "urgent threat" by Centers for Disease Control and Prevention (https://www.cdc. gov/drugresistance/biggest-threats.html). ${ }^{4}$ In the China Antimicrobial Surveillance Network (www.chinets.com/Chinet), clinical isolates of Acinetobacter in 2019 displayed resistance rates to cefoperazone-sulbactam 2:1, ampicillin-sulbactam, imipenem and meropenem of $46.5 \%, 69.3 \%, 73.6 \%$ and $75.1 \%$, respectively. 
Additionally, resistance rates to cefoperazone-sulbactam $1: 1$ and meropenem were $40.4 \%$ and $75.3 \%$, respectively, for Acinetobacter isolates collected from China in 20132014..$^{5}$ Despite potent in vitro antimicrobial activities, tigecycline and polymyxin demonstrate conspicuous disadvantages, such as poor distribution in tissue, low plasma concentration and obvious adverse reactions, which could preclude their clinical use. ${ }^{6}$ Hence, few treatment options are available against MDR $A$. baumannii.

Sulbactam, a semisynthetic penicillanic acid sulfone, acts as an inhibitor of $\beta$-lactamase and has intrinsic activity against the Acinetobacter genus. ${ }^{7}$ Previous study has found excellent antimicrobial activity of cefoperazone-sulbactam, ampicillin-sulbactam and carbapenemsulbactam combinations against MDR Acinetobacter in vitro and in vivo. ${ }^{8-11}$ In China, commercially available are sulbactam alone, ampicillin-sulbactam in proportion of $2: 1$ as well as cefoperazone-sulbactam in proportion of $2: 1$ and $1: 1$. As indicated by above resistance data, the current combinations could be insufficiently effective yet. Notably, adding more sulbactam to cefoperazonesulbactam combinations enhanced the in vitro antimicrobial activity against Acinetobacter. ${ }^{12}$ Consequently, sulbactam-based combinations, as one of alternative therapeutics, are attracting attention.

Therefore, we evaluated in vitro activity of ampicillinsulbactam, cefoperazone-sulbactam and imipenemsulbactam at different ratios against $A$. baumannii clinical isolates in China so as to find better antimicrobial regimens.

\section{Materials and Methods}

\section{Bacterial Strains}

A total of 300 clinical isolates of $A$. baumannii were collected from 29 hospitals in 26 cities across China in 2018, including carbapenem-susceptible $A$. baumannii (CSAB) and CRAB. They were isolated from respiratory tract $(199,66.3 \%)$, skin and skin structure $(21,7.0 \%)$, blood $(12,4.0 \%)$, urine tract $(8,2.7 \%)$, cerebrospinal fluid $(4,1.3 \%)$, bile $(3,1.0 \%)$ and others $(53,17.7 \%)$. Bacteria were preliminarily identified in the isolated hospitals, and confirmed by Matrix-assisted laser desorption ionization time-of-flight (MALDI-TOF) Vitek mass spectrometry (VMS) in our hospital. The study protocol was approved by the Institutional Review Board of Huashan Hospital, Fudan University (No. 2018-408).

\section{Antimicrobial Susceptibility Testing}

The minimum inhibitory concentrations (MICs) of antimicrobial agents were determined using broth microdilution method and interpreted on the basis of the Clinical and Laboratory Standards Institute (CLSI) criteria. $^{13}$ Sulbactam, $\beta$-lactam antibiotics, fluoroquinolones, amikacin, trimethoprim-sulfamethoxazole, tigecycline and polymyxin B were tested for comparison. Ampicillin, cefoperazone and imipenem were tested alone and in combination with sulbactam at the ratios of $2: 1,1: 1$, $1: 1.5,1: 2,1: 2.5$ and $1: 3$, respectively.

Given that neither CLSI nor European Committee on Antimicrobial Susceptibility Testing (EUCAST) provides the breakpoints for sulbactam-based combinations against A. baumannii, breakpoints for partner $\beta$-lactam antibiotics were referred carefully. ${ }^{12}$ Specifically, the MIC breakpoints for ampicillin and ampicillin-sulbactam were as follows: S, $\leq 8 \mathrm{mg} / \mathrm{L} ; \mathrm{I}, 16 \mathrm{mg} / \mathrm{L} ; \mathrm{R}, \geq 32 \mathrm{mg} / \mathrm{L}$. The MIC breakpoints for cefoperazone and cefoperazone-sulbactam were those for Enterobacteriaceae: S, $\leq 16 \mathrm{mg} / \mathrm{L}$; I, $32 \mathrm{mg} /$ $\mathrm{L} ; \mathrm{R}, \geq 64 \mathrm{mg} / \mathrm{L}$. The MIC breakpoints for imipenem and imipenem-sulbactam were as follows: $\mathrm{S}, \leq 2 \mathrm{mg} / \mathrm{L} ; \mathrm{I}, 4 \mathrm{mg} /$ $\mathrm{L} ; \mathrm{R}, \geq 8 \mathrm{mg} / \mathrm{L}$.

\section{Detection of $\beta$-Lactamase}

$\beta$-lactamase genes (bla $a_{\mathrm{TEM}}, b l a_{\mathrm{SHV}}, b l a_{\mathrm{VEB}}, b l a_{\mathrm{PER}}$, $b l a_{\mathrm{GES}}, b l a_{\mathrm{IMP}}, b l a_{\mathrm{VIM}}, b l a_{\mathrm{KPC}}, b l a_{\mathrm{OXA}-23}$ and $\left.b l a_{\mathrm{OXA}-58}\right)$ were detected by PCR. ${ }^{14}$ Primers sequences were obtained as previously described. ${ }^{13}$ Amplification was performed as follows: initial denaturation at $94^{\circ} \mathrm{C}$ for $5 \mathrm{~min}$; 30 cycles of denaturation at $94^{\circ} \mathrm{C}$ for $30 \mathrm{~s}$, annealing at $55^{\circ} \mathrm{C}$ for 30 $\mathrm{s}$ and elongation at $72^{\circ} \mathrm{C}$ for $1 \mathrm{~min}$; and a final elongation step at $72^{\circ} \mathrm{C}$ for $5 \mathrm{~min}$.

\section{Results}

\section{Antimicrobial Susceptibility Testing for Common Antibiotics and Identification of $\beta$-Lactamase}

Susceptibility rates of $300 \mathrm{~A}$. baumannii isolates to most comparator agents were less than $30 \%$, including $\beta$-lactams, fluoroquinolones, amikacin and trimethoprimsulfamethoxazole, while those to tigecycline and polymyxin B were $98.7 \%$ and $99.3 \%$, respectively (Table 1 ).

Among CRAB, 97.3\% (256/263) isolates harbored $b l a_{\mathrm{OXA}-23}$, and $b l a_{\mathrm{TEM}}$ was present in $51.7 \%(136 / 263)$ isolates. Neither $b l a_{\text {TEM }}$ nor $b l a_{\text {OXA-23 }}$ was detected in the CSAB isolates. None of the isolates carried $b l a_{\mathrm{SHV}}$, 
Table I Antimicrobial Susceptibilities of A. baumannii Determined by the Broth Microdilution Method

\begin{tabular}{|c|c|c|c|c|c|c|}
\hline \multirow[t]{2}{*}{ Antimicrobial Agents } & \multicolumn{3}{|c|}{ MIC (mg/L) } & \multirow{2}{*}{$\begin{array}{c}\text { Susceptible } \\
\text { Rate (\%) }\end{array}$} & \multirow{2}{*}{$\begin{array}{c}\text { Intermediate } \\
\text { Rate (\%) }\end{array}$} & \multirow{2}{*}{$\begin{array}{c}\text { Resistant } \\
\text { Rate (\%) }\end{array}$} \\
\hline & Range & $\mathrm{MIC}_{50}$ & $\mathrm{MIC}_{90}$ & & & \\
\hline Ceftazidime & $\mathrm{I}->32$ & $>32$ & $>32$ & 11.7 & 0.7 & 87.7 \\
\hline Cefepime & $0.5->128$ & 128 & $>128$ & 12.0 & 1.7 & 86.3 \\
\hline Aztreonam & $4->128$ & 64 & 128 & 3.3 & 7.7 & 89.0 \\
\hline Piperacillin-tazobactam ${ }^{a}$ & $\leq 2->256$ & $>256$ & $>256$ & 10.7 & 0.7 & 85.3 \\
\hline Meropenem & $0.12->64$ & 64 & $>64$ & 12.0 & 0.7 & 87.3 \\
\hline Ciprofloxacin & $\leq 0.06->8$ & $>8$ & $>8$ & 12.0 & 0.0 & 88.0 \\
\hline Levofloxacin & $\leq 0.12->16$ & 8 & $>64$ & 12.3 & 3.3 & 84.3 \\
\hline Amikacin & $\leq 1->128$ & $>128$ & $>128$ & 17.7 & 0.7 & 81.7 \\
\hline Trimethoprim-sulfamethoxazole ${ }^{b}$ & $\leq 0.25-32$ & $>32$ & $>32$ & 28.3 & l & 71.7 \\
\hline Tigecycline & $\leq 0.06-16$ & I & 2 & 98.7 & 1.0 & 0.3 \\
\hline Polymyxin B & $0.25-4$ & I & 2 & 99.3 & I & 0.7 \\
\hline
\end{tabular}

Notes: a For piperacillin-tazobactam, only concentration of piperacillin is listed. ${ }^{b}$ For trimethoprim-sulfamethoxazole, only concentration of trimethoprim is listed.

$b l a_{\mathrm{VEB}}, \quad b l a_{\mathrm{PER}}, \quad b l a_{\mathrm{GES}}, \quad b l a_{\mathrm{IMP}}, \quad b l a_{\mathrm{VIM}}, \quad b l a_{\mathrm{KPC}}$ or bla $a_{\text {OXA-58. }}$.

\section{Antimicrobial Susceptibility Testing for Sulbactam-Based Combinations}

In vitro activities of sulbactam, ampicillin, cefoperazone, imipenem and corresponding combinations are shown in Table 2. $\mathrm{MIC}_{50}$ and $\mathrm{MIC}_{90}$ values for sulbactam were $32 \mathrm{mg} / \mathrm{L}$ and $64 \mathrm{mg} / \mathrm{L}$, respectively. High resistant rates for ampicillin, cefoperazone and imipenem were observed (92.3\%, 93\% and $85.3 \%$, respectively). Overall, a stepwise increase in the ratio of sulbactam to $\beta$-lactams led to a stepwise decrease in the $\beta$-lactams MIC. Small-scale increase was observed in the susceptibility for imipenem-sulbactam from $13.7 \%$ to $16.3 \%$, while remarkable elevations were evidenced in the susceptibility for ampicillin-sulbactam and cefoperazone-sulbactam with the increasing proportion of sulbactam (from $12.3 \%$ to $58.3 \%$, from $15 \%$ to $91 \%$, respectively). Notably, susceptibility overtly increased from $29.3 \%$ for cefoperazone-sulbactam $1: 1$ to $66.3 \%$ for cefoperazone-sulbactam 1:1.5. Among all combinations, cefoperazone-sulbactam 1:3 demonstrated the most effective antibacterial activity with $\mathrm{MIC}_{90}$ of $16 \mathrm{mg} / \mathrm{L}$.

Although susceptibility rates of CSAB to ampicillin and cefoperazone were fairly low (only $10.8 \%$ and $18.9 \%$, respectively), all CSAB isolates were susceptible to ampicillin-sulbactam combinations and cefoperazonesulbactam combinations at all ratios (Table 3). For CRAB strains, escalating addition of sulbactam enhanced the in vitro antimicrobial activities of imipenemsulbactam, ampicillin-sulbactam and cefoperazone- sulbactam combinations gradually, with the susceptibility rate rising from $1.5 \%$ to $4.6 \%$, from $0 \%$ to $52.5 \%$, from $3.0 \%$ to $89.7 \%$, respectively.

\section{Discussion}

A. baumannii displayed high resistance rate to imipenem $(85.3 \%)$ in this study. Meanwhile, Acinetobacter spp. in the China Antimicrobial Surveillance Network in 2018 (www.chinets.com/Chinet) also depicted similar resistance rate to imipenem (73.2\%). A 10-year-spanning study in Hungary observed an increase in resistance levels of Acinetobacter spp. originating from urine samples. ${ }^{15}$ Although there are emerging novel antimicrobial agents for gram-negative bacteria, few of them are effective for A. baumannii yet. Hence, nowadays combination therapeutics based on existing drugs could be a practical approach to treating infections caused by $A$. baumannii.

This study demonstrated again that the higher ratio of sulbactam, the more potent in vitro antimicrobial activity of imipenem-sulbactam, ampicillin-sulbactam and cefoperazone-sulbactam combinations against A. baumannii. Higher susceptibility rate for cefoperazone-sulbactam was also observed compared with those of imipenemsulbactam and ampicillin-sulbactam. Cefoperazonesulbactam 1:3 displayed best in vitro activity among all sulbactam-based combinations and showed superior activity to most comparator agents, including other $\beta$-lactams, fluoroquinolones, aminoglycosides and trimethoprimsulfamethoxazole.

Previous study revealed that cefoperazone-sulbactam 1:1 exhibited greatest in vitro activity against CRAB, 
Table 2 In vitro Activities of Imipenem-Sulbactam, Ampicillin-Sulbactam and Cefoperazone-Sulbactam Against A. baumannii

\begin{tabular}{|c|c|c|c|c|c|c|}
\hline \multirow[t]{2}{*}{ Drugs } & \multicolumn{3}{|c|}{$\operatorname{MIC}(\mathrm{mg} / \mathrm{L})^{\mathrm{a}}$} & \multirow{2}{*}{$\begin{array}{c}\text { Susceptible } \\
\text { Rate (\%) }\end{array}$} & \multirow{2}{*}{$\begin{array}{c}\text { Intermediate } \\
\text { Rate (\%) }\end{array}$} & \multirow{2}{*}{$\begin{array}{c}\text { Resistant } \\
\text { Rate (\%) }\end{array}$} \\
\hline & Range & $\mathrm{MIC}_{50}$ & $\mathrm{MIC}_{90}$ & & & \\
\hline SUL & $0.5-256$ & 32 & 64 & I & I & l \\
\hline IPM & $0.12-128$ & 32 & 64 & 13.7 & 1.0 & 85.3 \\
\hline IPM-SUL 2:I & $0.12-256$ & 32 & 64 & 13.7 & 1.0 & 85.3 \\
\hline IPM-SUL I:I & $0.12-64$ & 16 & 32 & 14.3 & 1.0 & 84.7 \\
\hline IPM-SUL I:I.5 & $0.12-64$ & 16 & 32 & 14.3 & 1.3 & 84.3 \\
\hline IPM-SUL I:2 & $0.12-64$ & 8 & 16 & 15.0 & 3.7 & 81.3 \\
\hline IPM-SUL I:2.5 & $0.12-64$ & 8 & 16 & 15.7 & 9.0 & 75.3 \\
\hline IPM-SUL I:3 & $0.12-32$ & 8 & 16 & 16.3 & 18.3 & 65.3 \\
\hline AMP & $8->256$ & $>256$ & $>256$ & 1.3 & 6.3 & 92.3 \\
\hline AMP-SUL 2:I & $I->256$ & 64 & 128 & 12.3 & 2.0 & 85.7 \\
\hline AMP-SUL I:I & $0.5-256$ & 32 & 64 & 14.3 & 16.3 & 69.3 \\
\hline AMP-SUL I:I.5 & $0.25-256$ & 16 & 64 & 20.3 & 35.0 & 44.7 \\
\hline AMP-SUL I:2 & $0.25-256$ & 16 & 32 & 28.3 & 46.0 & 25.7 \\
\hline AMP-SUL I:2.5 & $0.12-128$ & 8 & 32 & 51.3 & 35.3 & 13.3 \\
\hline AMP-SUL I:3 & $0.25-128$ & 8 & 16 & 58.3 & 32.7 & 9.0 \\
\hline CFP & $16->256$ & $>256$ & $>256$ & 2.3 & 4.7 & 93.0 \\
\hline CFP-SUL 2:I & $I->256$ & 64 & 128 & 15.0 & 12.7 & 72.3 \\
\hline CFP-SUL I:I & $0.5->256$ & 32 & 64 & 29.3 & 47.3 & 23.3 \\
\hline CFP-SUL I:I.5 & $0.5-256$ & 16 & 32 & 66.3 & 24.0 & 9.7 \\
\hline CFP-SUL I:2 & $0.25-256$ & 16 & 32 & 78.7 & 14.0 & 7.3 \\
\hline CFP-SUL I:2.5 & $0.25-256$ & 16 & 32 & 86.3 & 8.0 & 5.7 \\
\hline CFP-SUL I:3 & $0.25-128$ & 8 & 16 & 91.0 & 4.3 & 4.7 \\
\hline
\end{tabular}

Notes: ${ }^{\text {F}}$ For MIC of IPM-SUL, AMP-SUL and CFP-SUL combinations, only the concentrations of imipenem, ampicillin and cefoperazone are listed respectively. Abbreviations: SUL, sulbactam; IPM, imipenem; IPM-SUL, imipenem-sulbactam; AMP, ampicillin; AMP-SUL, ampicillin-sulbactam; CFP, cefoperazone; CFP-SUL, cefoperazone-sulbactam.

Table 3 In vitro Activities of Ampicillin-Sulbactam and Cefoperazone-Sulbactam Against Carbapenem-Susceptible A. baumannii and Carbapenem-Resistant A. baumannii

\begin{tabular}{|c|c|c|c|c|c|c|c|}
\hline \multirow{2}{*}{$\begin{array}{l}\text { Drugs } \\
\text { CSAB }(n=37)\end{array}$} & \multicolumn{2}{|c|}{ MIC (mg/L) } & \multirow{2}{*}{ Susceptible Rate (\%) } & \multirow{2}{*}{$\begin{array}{l}\text { Drugs } \\
\text { CRAB }(n=263)\end{array}$} & \multicolumn{2}{|c|}{ MIC (mg/L) } & \multirow{2}{*}{ Susceptible Rate (\%) } \\
\hline & $\mathrm{MIC}_{50}$ & MIC $_{90}$ & & & $\mathrm{MIC}_{50}$ & $\mathrm{MIC}_{90}$ & \\
\hline SUL & I & 2 & l & SUL & 32 & 64 & l \\
\hline AMP & 16 & 32 & 10.8 & AMP & $>256$ & $>256$ & 0.0 \\
\hline AMP-SUL 2:I & 2 & 4 & 100.0 & AMP-SUL 2:I & 64 & 128 & 0.0 \\
\hline AMP-SUL I:I & I & 2 & 100.0 & AMP-SUL I:I & 32 & 64 & 2.3 \\
\hline AMP-SUL I:I.5 & 0.5 & I & 100.0 & AMP-SUL I:I.5 & 32 & 64 & 9.1 \\
\hline AMP-SUL I:2 & 0.5 & I & 100.0 & AMP-SUL I:2 & 16 & 32 & 18.3 \\
\hline AMP-SUL I:2.5 & 0.5 & 0.5 & 100.0 & AMP-SUL I:2.5 & 16 & 32 & 44.5 \\
\hline AMP-SUL I:3 & 0.25 & 0.5 & 100.0 & AMP-SUL I:3 & 8 & 32 & 52.5 \\
\hline CFP & 32 & 128 & 18.9 & CFP & $>256$ & $>256$ & 0.0 \\
\hline CFP-SUL 2:I & 2 & 4 & 100.0 & CFP-SUL 2:I & 64 & 128 & 3.0 \\
\hline CFP-SUL I:I & I & 2 & 100.0 & CFP-SUL I:I & 32 & 64 & 19.4 \\
\hline CFP-SUL I:I.5 & I & I & 100.0 & CFP-SUL I:I.5 & 16 & 64 & 61.6 \\
\hline CFP-SUL I:2 & 0.5 & I & 100.0 & CFP-SUL I:2 & 16 & 32 & 75.7 \\
\hline CFP-SUL I:2.5 & 0.5 & I & 100.0 & CFP-SUL I:2.5 & 16 & 32 & 84.4 \\
\hline CFP-SUL I:3 & 0.5 & 0.5 & 100.0 & CFP-SUL I:3 & 8 & 32 & 89.7 \\
\hline
\end{tabular}

Abbreviations: SUL, sulbactam; IPM, imipenem; IPM-SUL, imipenem-sulbactam; AMP, ampicillin; AMP-SUL, ampicillin-sulbactam; CFP, cefoperazone; CFP-SUL, cefoperazone-sulbactam; CSAB, carbapenem-susceptible A. baumannii; CRAB, carbapenem-resistant $A$. baumannii. 
followed by cefoperazone-sulbactam 2:1 and cefoperazone (susceptibility rate, $80.0 \%$ vs $40.0 \%$ and $0 \%$ ). ${ }^{16}$ Additionally, cefoperazone-sulbactam 1:2 demonstrated antimicrobial benefit, with the susceptibility rates for cefoperazone-sulbactam $1: 2,1: 1$ and $2: 1$ of $92.6 \%, 76.2 \%$ and $41.0 \%$, respectively. ${ }^{12}$ Similarly, imipenem-sulbactam at a 1:1 ratio was slightly superior to that at a $2: 1$ ratio (resistance rate, $23.3 \%$ vs $26.7 \%$ ) in inhibiting $\mathrm{CRAB}$ isolates. ${ }^{17}$ Our findings were basically consistent with these data, which revealed the importance of the ratio of sulbactam. However, there were scarce reports about in vitro activities of imipenem-sulbactam, ampicillinsulbactam combinations at higher ratios against A. baumannii clinical strains.

Growing clinical evidence has suggested sulbactam as a promising treatment option in the management of Acinetobacter infections. For the bloodstream infections caused by carbapenem-non-susceptible Acinetobacter spp., sulbactam-based regimens demonstrated similar clinical efficacy rates as carbapenem-based regimens $(50.0 \%$ vs $45.8 \%) .{ }^{18}$ In term of the treatment of XDR Acinetobacter infections, sulbactam-based regimens displayed higher clinical efficacy rate instead of carbapenem-based regimens $(62.5 \%$ vs $47.4 \%){ }^{19}$ A significant reduction in the 28 -day mortality was observed for those receiving cefoperazonesulbactam compared with those receiving tigecycline $(29.3 \%$ vs $51.9 \%){ }^{20}$ In a network meta-analysis, combination therapy of colistin with sulbactam was superior to that of colistin with tigecycline and colistin monotherapy in microbiological eradication while no significant differences were noted in all-cause mortality for MDR and XDR A. baumannii infections. ${ }^{21}$ Early study also reported lower incidence rate of adverse events of high-dose ampicillinsulbactam (30.7\%, including $15.3 \%$ nephrotoxicity) than that of colistin (39.6\%, including 33\% nephrotoxicity) in patients with MDR A. baumannii ventilator-associated pneumonia. $^{22}$ Additionally, compared with tigecycline and colistin, sulbactam is also characterized by advantages of tissue distribution, tolerability and cost.

CSAB displayed susceptibility to sulbactam alone, ampicillin-sulbactam combinations and cefoperazone-sulbactam combinations, and either $b l a_{\text {OXA23 }}$ or $b l a_{\text {TEM }}$ gene was undetected among them in this study. Sulbactam possesses the intrinsic activity against $A$. baumannii. It binds to penicillin-binding proteins (PBPs), hinders the synthesis of bacterial cell wall and kills bacteria. ${ }^{23}$ Moreover, sulbactam rendered the bacteria more susceptible to phagocytosis as well as to killing by polymorphonuclear leukocytes. ${ }^{24}$
Nevertheless, as indicated by in vitro activity, sulbactam alone was insufficient to fight against $\mathrm{CRAB}$, which could be primarily due to hydrolysis of $\beta$-lactamases, including class A $\beta$-lactamase TEM-1 and Class D $\beta$-lactamase OXA$23 .^{25-27}$ In fact, sulbactam activity could be potentiated by durlobactam (formerly ETX2514), ${ }^{28}$ a novel inhibitor for class $\mathrm{A}, \mathrm{C}$ and $\mathrm{D} \beta$-lactamases, with the combination sulbactam/durlobactam currently being assessed in a Phase III clinical trial in patients with A. baumannii infections (https:// clinicaltrials.gov/ct $2 /$ results? cond $=\&$ term $=$ ETX2514\&cntry $=\&$ state $=\&$ city $=\&$ dist $=$ ).

There are few studies on the actual mechanism underpinning enhanced antibacterial activity of $\beta$-lactamsulbactam combinations. The complementary and saturated PBP binding could be one of the mechanisms since $\beta$ lactams and sulbactam exhibit different binding affinities for PBPs. ${ }^{29,30}$ Another possible explanation could be the alternative shielding hypothesis. ${ }^{31}$ Sulbactam binds to the active site of the $\beta$-lactamases and acts as a better substrate for $\beta$-lactamases, thus preventing the hydrolysis of the partner $\beta$-lactam antibiotics and allowing the partner to reach its target PBPs more effectively. ${ }^{25-27,32}$ High turnover numbers of sulbactam for TEM-1 and OXA-23 enzyme (sulbactam/enzyme molar ratio required for complete inhibition of each enzyme) as well as the coexistence of other $\beta$-lactamases may rationalize high-ratio sulbactam in combinations. ${ }^{33}$ In addition, compounds could damage biofilm architecture significantly and exerted superior killing effects against CRAB. ${ }^{34}$ Overall, above hypotheses need further confirmations. However, it remains to explain why cefoperazone-sulbactam exhibited superior activity over imipenem-sulbactam and ampicillin-sulbactam.

Nevertheless, this study had some limitations. Firstly, numbers of clinical isolates were relatively small though these isolates were from multiple centers. Secondly, the $\beta$ lactamase type is relatively simple, focusing on TEM-1 and OXA-23. Further large-scale studies are warranted to verify our research.

\section{Conclusion}

In conclusion, the increasing proportion of sulbactam could enhance antimicrobial activity of imipenemsulbactam, ampicillin-sulbactam and cefoperazonesulbactam compound against $A$. baumannii strains, with cefoperazone-sulbactam as the most potent. Our study suggests that high-ratio cefoperazone-sulbactam compound could be a promising antimicrobial regimen for A. baumannii infection in China. 


\section{Acknowledgments}

This work was supported by grants from National Megaproject for Innovative Drugs (2019ZX09721001-006-004) and China Antimicrobial Surveillance Network (2018QD100). The funders had no role in study design, data collection and interpretation, or the decision to submit the work for publication. Part of this study was presented in the abstract book of 30th European Congress of Clinical Microbiology \& Infectious Diseases (ECCMID) in 2020.

\section{Disclosure}

The authors report no conflicts of interest in this work.

\section{References}

1. Douzandeh-Mobarrez B, Alizade H, Kafil HS, Karmostaji A. Antimicrobial categories in describing multidrug resistance, extensive drug resistance and pan-drug resistance in Pseudomonas aeruginosa and Acinetobacter baumannii: a systematic review. Rev Med Microbiol. 2021;32:6-11.

2. Aslam A, Gajdács M, Zin CS, Binti ARN, Ahmed SI, Jamshed SQ. Public awareness and practices towards self-medication with antibiotics among the Malaysian Population. A development of questionnaire and pilot-testing. Antibiotics. 2020;9:97.

3. Gajdács M, Urbán E. Epidemiological trends and resistance associated with Stenotrophomonas maltophilia bacteremia: a 10-year Retrospective Cohort Study in a tertiary-care hospital in Hungary. Diseases. 2019;7:41.

4. Shlaes DM, Bradford PA. Antibiotics-from there to where?: how the antibiotic miracle is threatened by resistance and a broken market and what we can do about it. Pathog Immun. 2018;3(1):19-43. doi:10.20411/pai.v3i1.231

5. Pfaller MA, Flamm RK, Duncan LR, Mendes RE, Jones RN, Sader HS. Antimicrobial activity of tigecycline and cefoperazone/sulbactam tested against 18,386 gram-negative organisms from Europe and the AsiaPacific region (2013-2014). Diagn Microbiol Infect Dis. 2017;88 (2):177-183. doi:10.1016/j.diagmicrobio.2017.02.020

6. Gajdács M. The concept of an ideal antibiotic: implications for drug design. Molecules. 2019;24:892. doi:10.3390/molecules24050892

7. Williams JD. Beta-lactamase inhibition and in vitro activity of sulbactam and sulbactam/cefoperazone. Clin Infect Dis. 1997;24:494-497. doi:10.1093/clinids/24.3.494

8. Higgins $P G$, Wisplinghoff $H$, Stefanik D, Seifert H. In vitro activities of the beta-lactamase inhibitors clavulanic acid, sulbactam, and tazobactam alone or in combination with beta-lactams against epidemiologically characterized multidrug-resistant Acinetobacter baumannii strains. Antimicrob Agents Chemother. 2004;48(5):1586-1592. doi:10.1128/AAC.48.5.1586-1592.2004

9. Ji J, Du X, Chen Y, Fu Y, Wang H, Yu Y. In vitro activity of sulbactam in combination with imipenem, meropenem, panipenem or cefoperazone against clinical isolates of Acinetobacter baumannii. Int $J$ Antimicrob Agents. 2013;41(4):400-401. doi:10.1016/j. ijantimicag.2012.12.014

10. Choi JY, Park YS, Cho CH, et al. Synergic in-vitro activity of imipenem and sulbactam against Acinetobacter baumannii. Clin Microbiol Infect. 2004;10(12):1098-1101. doi:10.1111/j.1469-0691.2004.00987.x

11. Lee NY, Wang CL, Chuang YC, et al. Combination carbapenem-sulbactam therapy for critically ill patients with multidrug-resistant Acinetobacter baumannii bacteremia: four case reports and an in vitro combination synergy study. Pharmacotherapy. 2007;27(11):1506-1511. doi:10.1592/phco.27.11.1506
12. Lai CC, Chen CC, Lu YC, Lin TP, Chuang YC, Tang HJ. Appropriate composites of cefoperazone-sulbactam against multidrug-resistant organisms. Infect Drug Resist. 2018;11:1441-1445. doi:10.2147/ IDR.S175257

13. CLSI. Performance Standards for Antimicrobial Susceptibility Testing. 30th ed. Wayne, PA: Clinical and Laboratory Standards Institute; 2020. CLSI supplement M100.

14. Dallenne C, Da CA, Decre D, Favier C, Arlet G. Development of a set of multiplex PCR assays for the detection of genes encoding important beta-lactamases in Enterobacteriaceae. J Antimicrob Chemother. 2010;65(3):490-495. doi:10.1093/jac/dkp498

15. Gajdács M, Burián K, Terhes G. Resistance levels and Epidemiology of Non-Fermenting Gram-Negative Bacteria in Urinary Tract Infections of inpatients and outpatients (RENFUTI): a 10-year epidemiological snapshot. Antibiotics. 2019;8:143.

16. Lai CC, Chen CC, Lu YC, Chuang YC, Tang HJ. In vitro activity of cefoperazone and cefoperazone-sulbactam against carbapenem-resistant Acinetobacter baumannii and Pseudomonas aeruginosa. Infect Drug Resist. 2019;12:25-29. doi:10.2147/IDR.S181201

17. Wang FD, Lin ML, Lee WS, Liu CY. In vitro activities of beta-lactam antibiotics alone and in combination with sulbactam against gram-negative bacteria. Int $J$ Antimicrob Agents. 2004;23 (6):590-595. doi:10.1016/j.jjantimicag.2003.10.008

18. Xu S, Li Y, Xu X, et al. A case-control study: clinical characteristics of nosocomial bloodstream infections versus non-bloodstream infections of Acinetobacter spp. Clin Infect Dis. 2018;67(suppl_2):S189S195. doi:10.1093/cid/ciy671

19. Li Y, Guo Q, Wang P, et al. Clonal dissemination of extensively drugresistant Acinetobacter baumannii producing an OXA-23 $\beta$-lactamase at a teaching hospital in Shanghai, China. J Microbiol Immunol Infect. 2015;48(1):101-108. doi:10.1016/j.jmii.2014.04.005

20. Niu T, Luo Q, Li Y, Zhou Y, Yu W, Xiao Y. Comparison of tigecycline or cefoperazone/sulbactam therapy for bloodstream infection due to carbapenem-resistant Acinetobacter baumannii. Antimicrob Resist Infect Control. 2019;8:52. doi:10.1186/s13756-019-0502-x

21. Kengkla K, Kongpakwattana K, Saokaew S, Apisarnthanarak A, Chaiyakunapruk N. Comparative efficacy and safety of treatment options for MDR and XDR Acinetobacter baumannii infections: a systematic review and network meta-analysis. $J$ Antimicrob Chemother. 2018;73(1):22-32. doi:10.1093/jac/dkx368

22. Betrosian AP, Frantzeskaki F, Xanthaki A, Douzinas EE. Efficacy and safety of high-dose ampicillin/sulbactam vs. colistin as monotherapy for the treatment of multidrug resistant Acinetobacter baumannii ventilator-associated pneumonia. $J$ Infect. 2008;56 (6):432-436. doi:10.1016/j.jinf.2008.04.002

23. Penwell WF, Shapiro AB, Giacobbe RA, et al. Molecular mechanisms of sulbactam antibacterial activity and resistance determinants in Acinetobacter baumannii. Antimicrob Agents Chemother. 2015;59 (3):1680-1689. doi:10.1128/AAC.04808-14

24. Kazmierczak A, Pechinot A, Siebor E, Cordin X, Labia R. Sulbactam: secondary mechanisms of action. Diagn Microbiol Infect Dis. 1989;12(4 Suppl):139S-146S. doi:10.1016/07328893(89)90126-0

25. Krizova L, Poirel L, Nordmann P, Nemec A. TEM-1 beta-lactamase as a source of resistance to sulbactam in clinical strains of Acinetobacter baumannii. J Antimicrob Chemother. 2013;68 (12):2786-2791. doi:10.1093/jac/dkt275

26. Yang $\mathrm{Y}, \mathrm{Xu} \mathrm{Q}, \mathrm{Li} \mathrm{T}$, et al. OXA-23 is a prevalent mechanism contributing to sulbactam resistance in diverse Acinetobacter baumannii clinical strains. Antimicrob Agents Chemother. 2019;63(1). doi:10.1128/AAC.01676-18

27. Kuo SC, Lee YT, Yang LT, et al. Contribution of Acinetobacter-derived cephalosporinase-30 to sulbactam resistance in Acinetobacter baumannii. Front Microbiol. 2015;6:231. doi:10.3389/fmicb.2015.00231 
28. Durand-Reville TF, Guler S, Comita-Prevoir J, et al. ETX2514 is a broad-spectrum beta-lactamase inhibitor for the treatment of drug-resistant gram-negative bacteria including Acinetobacter baumannii. Nat Microbiol. 2017;2:17104. doi:10.1038/ nmicrobiol.2017.104

29. Moya B, Barcelo IM, Bhagwat S, et al. Potent beta-lactam enhancer activity of zidebactam and WCK 5153 against Acinetobacter baumannii, including carbapenemase-producing clinical isolates. Antimicrob Agents Chemother. 2017;61(11). doi:10.1128/ AAC.01238-17

30. Papp-Wallace KM, Senkfor B, Gatta J, et al. Early insights into the interactions of different beta-lactam antibiotics and beta-lactamase inhibitors against soluble forms of Acinetobacter baumannii PBP1a and Acinetobacter sp. PBP3. Antimicrob Agents Chemother. 2012;56 (11):5687-5692. doi:10.1128/AAC.01027-12

31. Sutaria DS, Moya B, Green KB, et al. First penicillin-binding protein occupancy patterns of beta-lactams and beta-lactamase inhibitors in Klebsiella pneumoniae. Antimicrob Agents Chemother. 2018;62(6). doi:10.1128/AAC.00282-18
32. Wong D, Nielsen TB, Bonomo RA, Pantapalangkoor P, Luna B, Spellberg B. Clinical and pathophysiological overview of Acinetobacter infections: a century of challenges. Clin Microbiol Rev. 2017;30(1):409-447. doi:10.1128/CMR.00058-16

33. Shapiro AB. Kinetics of sulbactam hydrolysis by beta-lactamases, and kinetics of beta-lactamase inhibition by sulbactam. Antimicrob Agents Chemother. 2017;61(12). doi:10.1128/AAC.01612-17

34. Wang YC, Kuo SC, Yang YS, et al. Individual or combined effects of meropenem, imipenem, sulbactam, colistin, and tigecycline on biofilm-embedded Acinetobacter baumannii and biofilm architecture. Antimicrob Agents Chemother. 2016;60(8):4670-4676. doi:10.1128/AAC.00551-16
Infection and Drug Resistance

\section{Publish your work in this journal}

Infection and Drug Resistance is an international, peer-reviewed openaccess journal that focuses on the optimal treatment of infection (bacterial, fungal and viral) and the development and institution of preventive strategies to minimize the development and spread of resistance. The journal is specifically concerned with the epidemiology of
Dovepress

antibiotic resistance and the mechanisms of resistance development and diffusion in both hospitals and the community. The manuscript management system is completely online and includes a very quick and fair peerreview system, which is all easy to use. Visit http://www.dovepress.com/ testimonials.php to read real quotes from published authors. 